\title{
Effectiveness of percutaneous microelectrolysis and ultrasound in decreasing pain in myofascial trigger points: evaluation through algometry and visual analogue scale
}

\author{
DOI: https://doi.org/10.5114/pq.2020.95768 \\ Hernán Andrés de la Barra Ortiz', Jaime Opazo Cancino², Fernanda Sánchez Peña', Fernanda Soto León', \\ Edward Miño Donoso', Valeria Trecanao Gaete ${ }^{1}$ \\ ${ }^{1}$ Universidad Andres Bello, Santiago, Chile \\ 2 Universidad Diego Portales, Santiago, Chile
}

Abstract

Introduction. Percutaneous electrotherapy is a therapeutic alternative in musculoskeletal conditions. Percutaneous microelectrolysis (MEP) stands out as treatment for tendinopathies and musculoskeletal pain, such as myofascial trigger points (MTrPs), although more studies are needed to support it. The study aimed to investigate MEP effectiveness in reducing pain pressure threshold (PPT) and pain intensity (PI) in MTrPs.

Methods. A randomized controlled clinical trial was performed at Physiotherapy Laboratory of Andrés Bello University and involved 48 volunteers with MTrPs in upper trapezius muscle, randomly assigned to the experimental or control group. Both groups received baseline ultrasound treatment. In the experimental group, MEP intervention was applied with 3 re-evaluation sessions (on days 1, 3, and 7). Direct current was delivered with an acupuncture needle directly to MTrPs, and PPT and PI were re-evaluated before and after the application. The main outcomes were PPT and PI differences (PPTdiff and PIdiff) between evaluation sessions. Results. TMEP implied positive changes, increasing PPT (PPTdiff1-1, $p=0.0000$; PPTdiff2-1, $p=0.0000$; PPTdiff3-1, $p=0.0000$ ) and decreasing PI (PIdiff1-1, $p=0.0001$; Pldiff2-1, $p=0.0001$; Pldiff3-1, $p=0.0008$ ) in the experimental group. Significant differences were observed in PPT in the second re-evaluation session compared with the control group (PPTdiff2-1, $p=0.0032$ ).

Conclusions. MEP is a good analgesic treatment for MTrPs compared with therapeutic ultrasound, although neither of these therapies seems to be better than the other in a long term. They improve PPT and PI, so MEP can be considered effective alternative treatment for MTrPs pain.

Key words: electrotherapy, electrolysis, pain threshold, myofascial pain syndromes, ultrasound, visual analogue scale

\section{Introduction}

Electromedicine offers a diversity of therapeutic currents in rehabilitation today, although the lack of knowledge of many modalities has resulted in the use of the most traditional techniques, highlighting the application of sensitive transcutaneous electrical nerve stimulation (TENS) or median frequency currents for pain control, muscle strengthening, tissue healing, or oedema and inflammatory reactions management [1-3]. Among the oldest currents described is direct current (DC), or galvanic current, which stands out for its specific therapeutic effects that are not achieved with other electrical modalities. DC is a unidirectional and constant-intensity current [1]. Its biophysical characteristics facilitate accumulation of electrical charges in biological tissues near the point where electrodes are located, generating electrochemical changes. These effects have been called polar effects and are different for anode and cathode and would be responsible for physiological responses such as tissue $\mathrm{pH}$ changes, local circulatory modifications, or neuronal excitability changes [1, 4]. Human skin acts as a major obstacle to electrical current passage by accumulating electrical charges and increasing resistance. DC depth in cutaneous applications is up to $4 \mathrm{~cm}$, given the impedance increase of skin to unidirectional currents, but this depends on treatment time (minutes) and current density
$\left(\mathrm{mA} / \mathrm{cm}^{2}\right)$ [5-7]. The minimum and maximum recommended density for $D C$ is $0.05 \mathrm{~mA} / \mathrm{cm}^{2}$ and $1 \mathrm{~mA} / \mathrm{cm}^{2}$, respectively, with 10-15-minute treatment times and current densities not greater than $0.2 \mathrm{~mA} / \mathrm{cm}^{2}$ for electrodes. This recommendation is based on potential adverse effects that can generate electrochemical alkaline or acid burns [4, 5].

A relatively new technique in electrotherapy using $D C$ is percutaneous microelectrolysis (MEP), a procedure that has attracted great interest of several physiotherapists in recent years owing to its clinical results $[7,8]$. MEP constitutes a minimally invasive procedure in which low-intensity DC (micro galvanic current up to $0.96 \mathrm{~mA}$ ) is delivered by using acupuncture needles, generating a high current density (about $3.8 \mathrm{~mA} / \mathrm{cm}^{2}$ ) [8]. MEP induces a controlled local inflammatory process that promotes tissue repair accompanied by analgesic effects. These effects are supported by electrochemical changes (polar effects) that occur at the site of needle introduction, and with the intervention being a microcurrent applied through a small-sized electrode (needle), no important nociceptive responses are produced [1, 8, 9].

MEP uses polar effects of cathode through an acupuncture needle, producing release of sodium hydroxide, which increases tissue $\mathrm{pH}$. This reaction is the result of sodium ions $\left(\mathrm{Na}^{2+}\right)$ and water molecules $\left(\mathrm{H}_{2} \mathrm{O}\right)$ interaction, forming sodium hydroxide $(\mathrm{NaOH})$ and hydrogen $\left(\mathrm{H}^{+}\right)$as products.

Correspondence address: Hernán Andrés de la Barra Ortiz, Universidad Andrés Bello, Facultad de Ciencias de la Rehabilitación, Escuela de Kinesiología, Avenida Fernández Concha 700, Las Condes, Región Metropolitana, Chile, e-mail: hdelabarra@unab.cl or handresdelabarra@yahoo.es 
Sodium hydroxide is an alkaline caustic compound that generates thermal ablation $[4,8]$. It is also reported that the caustic reaction would maintain the analgesic effect by destroying local free nerve endings that process nociceptive information. Furthermore, the mechanical stimulus of the needle generates a micro tissue break, accompanied by physiological effects of electrical stimulus [8, 10].

MEP is used today in the traumatological and sports field as a treatment for muscular and tendinous injuries, and in the dermatofunctional area for wrinkles, stretch marks, fibrosis, and neuropathic scars management. MEP generates new collagen synthesis, followed by tissue repair process and inflammatory response induced by microelectrolysis $[8,10,11]$. The intervention has also been proposed for myofascial trigger points (MTrPs) treatment owing to good therapeutic response obtained in other tissues, although more research is needed [8]. MTrPs are tender and/or painful points located in muscle or myotendinous junction, which are felt as painful hard bands and can produce central nervous system excitation, generating referred pain. In addition, patients present rigidity, subjective weakness, movement pain, and slight decreases in mobility range [12].

The cause for MTrPs has not been clarified, although it is postulated that they can be generated by overload, stress, fatigue, direct trauma, cooling, visceral diseases, arthritic joints, and emotional tension [12, 13]. MTrPs present no signs of neurological deficits or haematological, biochemical, or urinary laboratory tests alterations, except in cases of concomitant diseases such as nutritional or metabolic conditions [13]. In addition, imaging examinations as X-rays or magnetic resonance do not reveal any pathological changes in the affected muscle or connective tissue $[14,15]$.

MTrPs can be diagnosed by physical examination with certain clinical criteria, which include a palpable, taut band; discomfort with local pressure at a muscle point, which may or may not generate referred pain; full muscle stretch mobility limitation; and individual self-recognition sensibility [15].

Pain pressure threshold (PPT) is one-way evaluation that allows to analyse painful points, providing a quantitative sensitivity assessment. It is commonly utilized in evaluating MTrPs in myofascial syndromes or hyperalgesia [16].

Algometry is a validated measurement used to assess PPT, which allows maximum tolerance pressure testing [17-19]. An algometer is an instrument that evaluates PPT by applying perpendicular pressure to the area of maximum sensitivity. The measurement unit in algometry is $\mathrm{kg} / \mathrm{cm}^{2}$ or newton $[16,17,19]$. Algometry is also used to measure pain in MTrPs. The assessment is considered positive if there exists a difference in pressure of $2 \mathrm{~kg} / \mathrm{cm}^{2}$ or more as related to a normal control point, usually measured at the opposite side. A PPT less than $3 \mathrm{~kg} / \mathrm{cm}^{2}$ is also considered positive [20, 21].

Pain intensity $(\mathrm{PI})$ is one of the most common measures used to assess pain magnitude. There are many instruments to determine PI; the visual analogue scale (VAS) is an easily applied, low-cost tool [22]. VAS consists of a $10-\mathrm{cm}$ transverse line that represents pain severity (continuous spectrum of painful experience). The 'painless' and 'worst pain' qualifiers are represented at the line ends. The patient is asked to make a mark at a point in the line which represents their pain at that moment. The evaluator records the response by measuring the millimetres that reflect PI [23].

In this context, the general objective of this research was to assess the effects of MEP in reducing pain in MTrPs, considering PPT and PI as primary variables.

\section{Subjects and methods}

\section{Design}

A randomized double-blind clinical trial was performed. All participants were diagnosed with MTrPs in shorter upper trapezius muscle. PPT and PI were recorded before and after the intervention. The patients were divided into 2 groups; both groups received therapeutic ultrasound as baseline treatment. In the experimental group, microelectrolysis was applied additionally. A reassessment of PPT and PI was carried out on days 1,3 , and 7 after the intervention.

\section{Subjects}

Overall, 48 healthy volunteers were recruited (23 men, 25 women; average age: 22 years). The participants were students of Rehabilitation Science Faculty (RSF) of Andrés Bello University, Santiago, Chile. Their number was selected by convenience of the researchers. The invitation was provided to students through formal communication channels (mailing), student representatives, and social networks, summoning 207 potential participants. The selection was based on a survey whose first part was structured in relation to the participant's general demographic data, including name, age, sex, body mass index (BMI), career year, and personal information contact (e-mail and cell phone number). The second part of the survey consisted of closed questions referring to the eligibility criteria. The inclusion criteria involved age over 18 years, being a student of RSF, and positive MTrPs. The exclusion criteria were neck or shoulder pathologies within the previous 6 months (such as fractures, sprains, tendinopathies, dislocations, or muscle tears), cervical pain, sensitivity alterations such as hypoesthesia, anaesthesia or hyperesthesia in the neck, shoulders, and/or arms, wounds or skin alterations in shoulder such as psoriasis, scars, or keloids, fear of electrotherapy application and analgesic pharmacological treatment at the recruitment time (non-steroidal anti-inflammatory or steroidal drugs). The elimination criteria involved non-tolerance to electrotherapy intervention and non-completion of the evaluation protocol (attendance at all scheduled sessions). Demographic data (Table 1) were tabulated with the use of Microsoft Excel ${ }^{\circledR} 2016$ software.

Secondary variables, such as short upper trapezius muscle laterality (SUTL) and sex, were represented as frequencies, while MTrP location distance and age were represented as median and BMI in averages. Primary variables included pre-intervention PPT and PI (PPTpre and PIpre) and were expressed as averages with their corresponding standard deviations $(S D)$. Data were analysed to compare the homogeneity of groups with the Stata v. 13 software.

\section{Equipment}

A Gymna Combi 500 electric stimulator was used for ultrasound application at $1 \mathrm{MHz}, 1.5 \mathrm{~W} / \mathrm{cm}^{2}, 100 \%$ duty cycle, $5 \mathrm{~cm}^{2}$ effective radiation area (ERA), and 15 minutes [24]. Sveltia DC equipment was used for MEP application. A dispersing electrode (area: $\left.28.26 \mathrm{~cm}^{2}\right)$ and an acupuncture needle $(0.3$ $\times 2.5 \mathrm{~cm}$ ) were utilized. The circuit was closed by placing the dispersing electrode in the arm contralateral to the trapezius muscle to be treated. Current intensity was $0.6 \mathrm{~mA}$ at the needle (current density: $1.71 \mathrm{~mA} / \mathrm{cm}^{2}$ ). 
Table 1. Characteristics of the study groups

\begin{tabular}{|c|c|c|c|c|}
\hline Variable & $\begin{array}{l}\text { Experimental group } \\
\qquad(n=24)\end{array}$ & $\begin{array}{c}\text { Control group } \\
\quad(n=24)\end{array}$ & $p$ & Sample distribution \\
\hline \multicolumn{5}{|l|}{$\operatorname{Sex}(\%)$} \\
\hline Men & $11(22.9)$ & $12(25.0)$ & \multirow{2}{*}{$0.7730^{*}$} & \multirow{2}{*}{ Normal $^{\star}$} \\
\hline Women & $13(27.1)$ & $12(25.0)$ & & \\
\hline Age $($ mean $\pm S D)$ & $22.5 \pm 1.9$ & $22.0 \pm 1.6$ & $0.2993^{\star *}$ & Normal $^{\star *}$ \\
\hline $\operatorname{BMI}\left(\mathrm{kg} / \mathrm{m}^{2}\right)($ mean $\pm S D)$ & $24.5 \pm 3.4$ & $22.9 \pm 2.3$ & $0.0616^{* *}$ & Normal $^{* *}$ \\
\hline \multicolumn{5}{|l|}{ Short trapezius muscle laterality (\%) } \\
\hline Right & $17(70.8)$ & $20(83.3)$ & \multirow{2}{*}{$0.2470^{\star \star \star}$} & \multirow{2}{*}{ Normal ${ }^{* \star *}$} \\
\hline Left & $7(29.2)$ & $4(16.7)$ & & \\
\hline $\mathrm{SP}-\mathrm{AC}$ distance $(\mathrm{cm})($ mean $\pm S D)$ & $9.2 \pm 1.8$ & $8.4 \pm 1.7$ & $0.1241^{\star *}$ & Normal $^{\star *}$ \\
\hline PPTpre1 $\left(\mathrm{kg} / \mathrm{cm}^{2}\right)($ mean $\pm S D)$ & $1.4 \pm 0.2$ & $1.4 \pm 0.2$ & $0.5734^{\star \star}$ & Normal $^{\star \star}$ \\
\hline Plpre1 (mm) (mean $\pm S D)$ & $32.1 \pm 13.6$ & $31.4 \pm 15.1$ & $0.8729^{\star *}$ & Normal $^{\star *}$ \\
\hline
\end{tabular}

The variables of sex and short trapezius muscle laterality are represented in frequencies (\%). Values for continuous variables are in means with their corresponding standard deviations $(S D)$.

* The variable of sex was analysed with the chi-square test.

${ }^{* *}$ For the analysis of continuous variables, Student's $t$-test was used.

*** The variable of short trapezius laterality was analysed with Fisher's $F$-test.

$\mathrm{BMI}$ - body mass index, SP-AC distance - distance between spinous process from C7 to acromion, PPTpre1 - pain pressure threshold before intervention on day 1, Plpre1 - pain intensity before intervention on day 1

\section{Measurement tools}

Baseline ${ }^{\circledR}$ pressure algometer was used to measure PPT ( $1 \mathrm{~cm}^{2}$ surface area) [25]. Algometry was applied after short upper trapezius assessment by placing the algometer at the MTrP and exerting perpendicular pressure until the patient reported pain. Then, the PPT value was registered $\left(\mathrm{kg} / \mathrm{cm}^{2}\right)$. PI was evaluated with VAS after algometry, recording pain perceived with the algometry test.

\section{Procedure}

\section{Participants}

Overall, 207 surveys with selection criteria questions were analysed, providing 59 potential participants. Out of these, 5 gave up once they were summoned; 54 agreed to participate and gave their written consent. Six participants were excluded owing to absence of MTrPs, which resulted in 48 patients (23 men, 25 women) (Figure 1). The principal investigator assigned a number to each participant to perform randomization. Group allocation was random and carried out by a simple randomization process with the use of dark envelopes. The participants did not know their assignment and were individually invited in the following 2 weeks to the Physiotherapy Laboratory of RSF for evaluation.

\section{Groups}

The sample was divided by a simple randomization process performed with a random number table, which assigned the participants to 1 of 2 study groups. The sequence of randomization and participants of each group was only known by the principal investigator. The groups were designed as the experimental (MEP group, $n=24$ ) and control group ( $n=$ 24). No subjects knew the treatment that would be applied. Each participant was evaluated for age, BMI, and SUTL.
MTrP location was determined at short trapezius muscle, with the reference of a line between superior acromion midpoint surface and $\mathrm{C} 7$ spinous process. Algometry and VAS were subsequently applied to obtain pre-intervention PPT and PI values (PPTpre and Plpre). The described variables were compared to assess the homogeneity of groups (Table 1). The data reflect the homogeneity of groups at the beginning of the study.

\section{Pain pressure threshold and pain intensity pre-intervention evaluation}

A blinded physiotherapist registered PPT and PI before and after the intervention. The evaluation station consisted of a chair and a table with an algometer and VAS. The subjects sat with their backs supported, with both feet on the floor. The evaluator proceeded to measure both upper trapezius muscles length manually, choosing the shortest one. Subsequently, a line was drawn between the $\mathrm{C} 7 \mathrm{spi}$ nous process and upper acromion midpoint face of the ipsilateral shoulder. The most sensitive palpation point was searched on the line and marked with a cross, with measuring the distance from $\mathrm{C} 7$ to this point. Algometry was applied at the selected point by exerting perpendicular pressure until the patient referred pain. The participants were instructed to slightly raise the contralateral hand when feeling pain in the algometry test. PPT less than $3 \mathrm{~kg} / \mathrm{cm}^{2}$ was recorded as a positive MTrP. The same protocol was used as for participant's pre-selection where 6 of 54 were excluded. Next, PI was assessed with VAS, recording pain magnitude $(\mathrm{mm})$ produced by algometry. PPTpre and PIpre data were tabulated in a Microsoft Excel ${ }^{\circledR}$ sheet.

\section{Therapeutic ultrasound application}

The participants of both groups received therapeutic ultrasound treatment. The experimental group was managed 


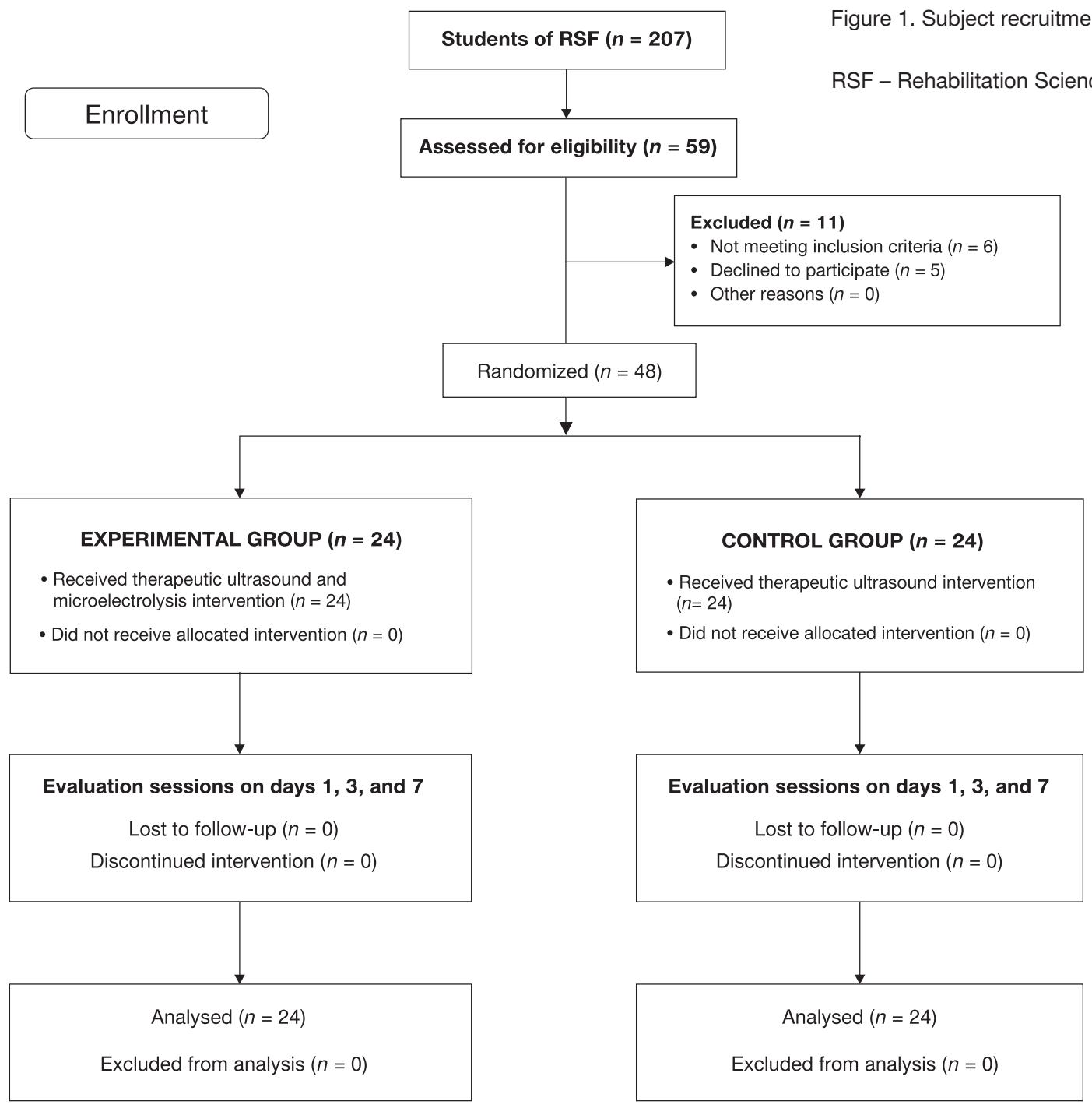

with ultrasound first and subsequently with MEP, while the control group was treated only with ultrasound. The ultrasound intervention protocol was performed in the same way for both groups, in an adjoining room, by another physiotherapist, who did not know group allocation. The treatment was carried out with the participant in a sitting position, by applying ultrasound at the sensitive point marked by the evaluation station examiner. The ultrasound therapy was delivered at $1 \mathrm{MHz}, 1.5 \mathrm{~W} / \mathrm{cm}^{2}, 100 \%$ duty cycle (spatial average temporal peak [SATP]: $1.5 \mathrm{~W} / \mathrm{cm}^{2}$ and spatial average temporal average [SATA]: $1.5 \mathrm{~W} / \mathrm{cm}^{2}$ ), ERA $5 \mathrm{~cm}^{2}$, and $15 \mathrm{~min}-$ utes [24]. Once the ultrasound intervention was finished, the MEP group participants were taken to the microelectrolysis box, while those of the control group returned to the evaluation station.

\section{Microelectrolysis application}

Microelectrolysis was performed by another physiotherapist. Before the intervention, the puncture area was cleaned with alcohol. The procedure was carried out with latex gloves. An acupuncture needle was introduced perpendicularly to the demarcated point of trapezius with $0.14 \mathrm{~mA}$ intensity. The dispersing electrode was placed on the external surface of the arm contralateral to the chosen trapezius muscle. Inside-tissue intensity was raised to $0.6 \mathrm{~mA}$. Each participant was instructed to report to the physiotherapist any to pause emission. Emission time was recorded until symptoms appeared, indicating T1 time, pausing emission for 30 seconds. Then, the second emission was made until the participant again referred discomfort symptoms. Second emission time was recorded as T2, pausing application for 30 seconds again. The third and last emission was delivered for the time obtained for T2 or until discomfort if it appeared before the time completion.

\section{Pain pressure threshold and pain intensity} post-intervention evaluation

After the intervention, the participants returned to the evaluation station. The evaluator repeated the same assessment protocol as before ultrasound and MEP application, recording post-intervention PPT and PI. The PPTdiff1-1 and Pldiff1-1 variables were based on differences between PPT and PI measurements before and after the intervention. The subjects were invited for re-evaluation on days 3 and 7 to obtain the variables of PPT2, PI2, PPT3, and PI3. This allowed to create the PPTdiff2-1, PPTdiff3-1, PIdiff2-1, and PIdiff3-1 variables, representing differences between the measurements obtained on days 3 and 7 and those obtained on day 1 before the intervention.

\section{Ethical approval}

The research related to human use has complied with all the relevant national regulations and institutional policies, has 
followed the tenets of the Declaration of Helsinki, and has been approved by Santiago Eastern Metropolitan Health Service (SSMO) ethics committee (June 18, 2019). The study was registered at www.clinicaltrials.gov under the ID of NCT03636386.

\section{Informed consent}

Informed consent has been obtained from all individuals included in this study.

\section{Results}

The sample was categorized with regard to age, sex, BMI, SUTL, PPTpre, and PIpre. Shapiro-Wilk test was applied to analyse normal distribution and then compare homogeneity between groups (Table 1). The statistical analysis performed in relation to primary and secondary variables did not show any statistically significant differences, so the groups were comparable at the beginning of the study.

Table 2 shows the evaluation results obtained for PPTpre, PPTpost, PPT2, PPT3, PPTdiff1-1, PPTdiff2-1, PPTdiff3-1,
Plpre, Plpost, PI2, PI3, Pldiff1-1, Pldiff2-1, and PIdiff3-1 for each group. Averages with respective standard deviations $(S D)$ were determined for all variables. Shapiro-Wilk test showed normal distribution, so Student's $t$-test was used. PPTdiff1-1 equalled $0.2 \pm 0.2$ for the MEP group and $0.2 \pm 0.1$ for the control, with no statistically significant difference between the groups $(p=0.0520)$. Mean PPTdiff2- 1 was $0.4 \pm 0.2$ for the MEP group and $0.2 \pm 0.2$ for the control, showing statistically significant differences in favour of the experimental group ( $p=0.0032$ ). Mean PPTdiff3-1 was $0.5 \pm 0.4$ for the MEP group and $0.3 \pm 0.3$ for the control, without differences between the groups $(p=0.0548)$. Pldiff1-1 equalled $-7.5 \pm 8.4$ for the MEP group and $-6.6 \pm 8.6$ for the control, without any statistically significant difference between the groups ( $p=0.3557)$. Mean Pldiff2- 1 was $-10.5 \pm 11.9$ for the MEP group and $-7.6 \pm 12.1$ for the control, with no statistically significant differences ( $p=0.2055)$. Mean PPTdiff3-1 was $-14.0 \pm 12.3$ for the MEP group and $-11.5 \pm 13.1$ for the control, without any difference between the groups $(p=0.2457)$.

Table 3 presents the PPTdiff and PIdiff between days 1 , 3, and 7. Shapiro-Wilk test showed normal distribution, so Stu-

Table 2. Pain pressure threshold and pain intensity differences between the groups

\begin{tabular}{|c|c|c|c|c|c|c|c|}
\hline Variable & $\begin{array}{c}\text { Experimental } \\
\text { group } \\
(n=24)\end{array}$ & $\begin{array}{l}\text { Control } \\
\text { group } \\
(n=24)\end{array}$ & $p$ & Variable & $\begin{array}{c}\text { Experimental } \\
\text { group } \\
(n=24)\end{array}$ & $\begin{array}{l}\text { Control } \\
\text { group } \\
(n=24)\end{array}$ & $p$ \\
\hline PPTpost $\left(\mathrm{kg} / \mathrm{cm}^{2}\right)($ mean $\pm S D)$ & $1.6 \pm 0.2$ & $1.6 \pm 0.2$ & 0.4008 & Plpost $(\mathrm{mm})($ mean $\pm S D)$ & $24.6 \pm 12.7$ & $24.8 \pm 15.1$ & 0.9508 \\
\hline PPT2 $\left(\mathrm{kg} / \mathrm{cm}^{2}\right)($ mean $\pm S D)$ & $1.7 \pm 0.1$ & $1.6 \pm 0.2$ & $0.0062^{*}$ & $\mathrm{Pl} 2(\mathrm{~mm})($ mean $\pm S D)$ & $21.6 \pm 11.0$ & $23.8 \pm 14.0$ & 0.5461 \\
\hline PPT3 $\left(\mathrm{kg} / \mathrm{cm}^{2}\right)($ mean $\pm S D)$ & $1.9 \pm 0.3$ & $1.7 \pm 0.2$ & 0.0805 & $\mathrm{PI} 3(\mathrm{~mm})($ mean $\pm S D)$ & $18.1 \pm 9.9$ & $20.0 \pm 13.2$ & 0.5815 \\
\hline PPTdiff1-1 $\left(\mathrm{kg} / \mathrm{cm}^{2}\right)($ mean $\pm S D)$ & $0.2 \pm 0.2$ & $0.2 \pm 0.1$ & 0.0520 & Pldiff1-1 $(\mathrm{mm})($ mean $\pm S D)$ & $-7.5 \pm 8.4$ & $-6.6 \pm 8.6$ & 0.3557 \\
\hline PPTdiff2-1 $\left(\mathrm{kg} / \mathrm{cm}^{2}\right)($ mean $\pm S D)$ & $0.4 \pm 0.2$ & $0.2 \pm 0.2$ & $0.0032^{*}$ & Pldiff2-1 $(\mathrm{mm})($ mean $\pm S D)$ & $-10.5 \pm 11.9$ & $-7.6 \pm 12.1$ & 0.2055 \\
\hline PPTdiff3-1 $\left(\mathrm{kg} / \mathrm{cm}^{2}\right)($ mean $\pm S D)$ & $0.5 \pm 0.4$ & $0.3 \pm 0.3$ & 0.0548 & Pldiff3-1 $(\mathrm{mm})($ mean $\pm S D)$ & $-14.0 \pm 12.3$ & $-11.5 \pm 13.1$ & 0.2457 \\
\hline
\end{tabular}

PPT, PI, PPTdiff, and PIdiff variables measured by groups after baseline ultrasound treatment and MEP intervention for all evaluation sessions. Values are means with their corresponding standard deviations $(S D)$. Data analysed with the parametric Student's $t$-test to compare intergroup differences for variables.

PPT - pain pressure threshold, PI - pain intensity, pre - pre-intervention, post - post-intervention, PPTdiff1-1 - PPT difference between pre- and post-intervention on day 1, PPTdiff2-1 - PPT difference between pre-intervention on day 1 and the second evaluation on day 3 , PPTdiff3-1 - PPT difference between pre-intervention on day 1 and the third evaluation on day 7, Pldiff1-1 - PI difference between pre- and post-intervention on day 1, Pldiff2-1 - PI difference between pre-intervention on day 1 and the second evaluation on day 3, Pldiff3-1 - PI difference between pre-intervention on day 1 and the third evaluation on day 7

${ }^{*} p<0.05$

Table 3. Pain pressure threshold and pain intensity differences between sessions for the MEP and control groups separately

\begin{tabular}{|l|c|c|c|}
\hline Variable & $\begin{array}{c}\text { Experimental group } \\
(n=24)\end{array}$ & $p$ & $\begin{array}{c}\text { Control group } \\
(n=30)\end{array}$ \\
\hline PPTdiff1-1 $\left(\mathrm{kg} / \mathrm{cm}^{2}\right)($ mean $\pm S D)$ & $0.2 \pm 0.2$ & $0.0000^{*}$ & $0.2 \pm 0.1$ \\
\hline PPTdiff2-1 $\left(\mathrm{kg} / \mathrm{cm}^{2}\right)($ mean $\pm S D)$ & $0.4 \pm 0.2$ & $0.0000^{*}$ & $0.2 \pm 0.2$ \\
\hline PPTdiff3-1 $\left(\mathrm{kg} / \mathrm{cm}^{2}\right)($ mean $\pm S D)$ & $0.5 \pm 0.4$ & $0.0000^{*}$ & $0.3 \pm 0.3$ \\
\hline Pldiff1-1 $(\mathrm{mm})(\mathrm{mean} \pm S D)$ & $-8.0 \pm 8.6$ & $0.0001^{*}$ & $-6.6 \pm 8.6$ \\
\hline Pldiff2-1 $(\mathrm{mm})$ (mean $\pm S D)$ & $-10.8 \pm 12.2$ & $0.0001^{*}$ & $-7.6 \pm 12.1$ \\
\hline Pldiff3-1 $(\mathrm{mm})$ (mean $\pm S D)$ & $-14.3 \pm 14.5$ & $0.0000^{*}$ & $0.0008^{*}$ \\
\hline
\end{tabular}

Variables measured by groups after baseline ultrasound treatment and MEP intervention for all evaluation sessions. Values are means with their corresponding standard deviations $(S D)$. Data analysed with the parametric Student's $t$-test to compare intragroup PPT and PI differences. A statistically significant difference for each variable was shown when groups were analysed individually.

PPTdiff1-1 - PPT difference between pre- and post-intervention on day 1, PPTdiff2-1 - PPT difference between pre-intervention on day 1 and the second evaluation on day 3, PPTdiff3-1 - PPT difference between pre-intervention on day 1 and the third evaluation on day 7 , PIdiff1-1 - PI difference between pre- and post-intervention on day 1, Pldiff2-1 - PI difference between pre-intervention on day 1 and the second evaluation on day 3, PIdiff3-1 - PI difference between pre-intervention on day 1 and the third evaluation on day 7 ${ }^{*} p<0.05$ 


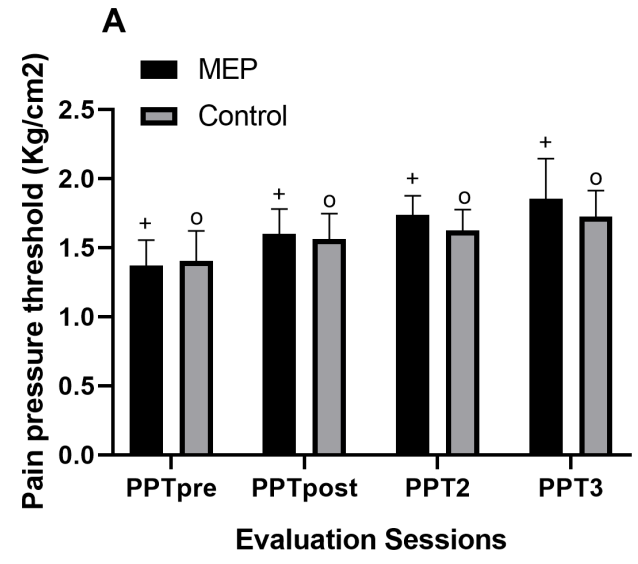

C

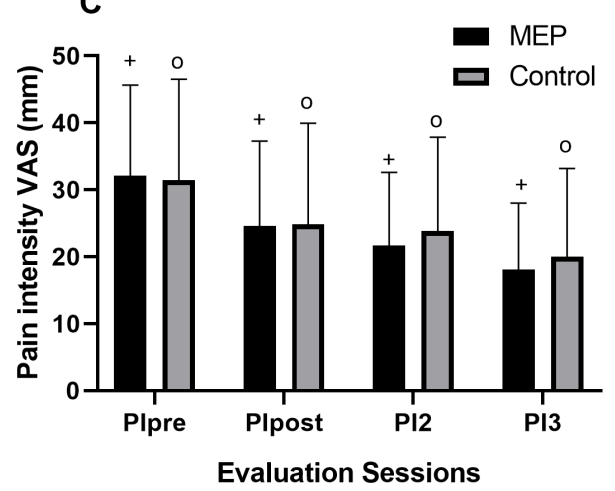

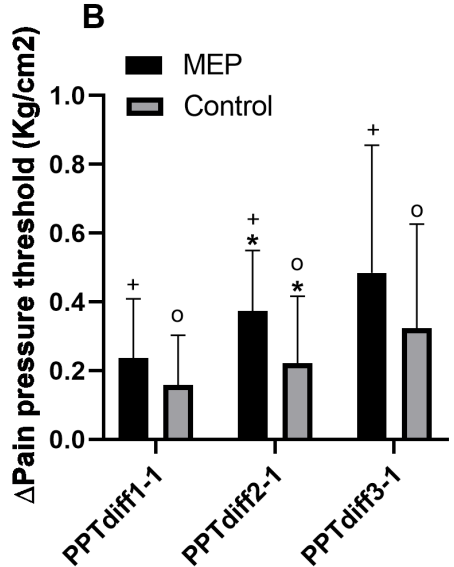

Evaluation Sessions

D

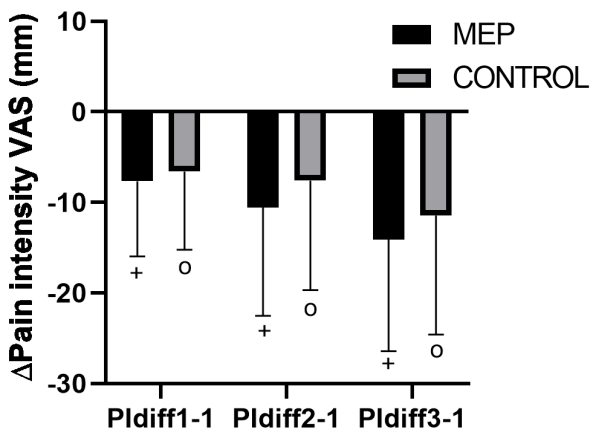

Evaluation Sessions

MEP - percutaneous microelectrolysis, VAS - visual analogue scale, PPT - pain pressure threshold, PI - pain intensity, pre - pre-intervention, post - post-intervention, PPTdiff1-1 - PPT difference between pre- and post-intervention on day 1, PPTdiff2-1 - PPT difference between pre-intervention on day 1 and the second evaluation on day 3, PPTdiff3-1 - PPT difference between pre-intervention on day 1 and the third evaluation on day 7, PIdiff1-1 - PI difference between pre- and post-intervention on day 1, Pldiff2-1 - PI difference between pre-intervention on day 1 and the second evaluation on day 3, Pldiff3-1 - PI difference between pre-intervention on day 1 and the third evaluation on day 7

Figure 2. MEP and ultrasound therapy improved PPT $(A, B)$ and $P I(C, D)$ in both groups. A statistically significant difference

$\left({ }^{*} p<0.05\right)$ for PPTdiff2-1 was observed between groups in the second evaluation. $(A, B)$ Intragroup analysis for the MEP (+) and control (o) groups shows significant improvements after intervention for PPTdiff1-1, PPTdiff2-1, and PPTdiff3-1. (C, D) Intragroup analysis for the MEP (+) and control (o) groups shows significant improvements after intervention for Pldiff1-1, PIdiff2-1, and PIdiff3-1. MEP group: $n=24$, control group: $n=24$

dent's $t$-test was used. Significant differences were observed in the MEP group for PPTdiff1-1 $(p=0.0000)$, PPTdiff2-1 $(p=$ $0.0000)$, PPTdiff3-1 $(p=0.0000)$, Pldiff1-1 $(p=0.0001)$, Pldiff2-1 ( $p=0.0001)$, and PIdiff3-1 $(p=0.0008)$. There were also statistically significant differences for the control group in the variables of PPTdiff1-1 $(p=0.0001)$, PPTdiff2-1 $(p=$ $0.0000)$, PPTdiff3-1 ( $p=0.0002)$, Pldiff1-1 $(p=0.0022)$, PIdiff2-1 ( $p=0.0008)$, and Pldiff3-1 ( $p=0.0001)$.

Figure 2 represents PPT, PPTdiff, PI, and PIdiff for sessions on days 1, 3, and 7 for each group. Statistically significant differences were observed in the second evaluation session between the MEP and the control group. In both groups, there were significant differences for PPTdiff and PIdiff when analysed independently, visualizing an improvement in pressure tolerance and decrease in PI between the evaluation sessions.

\section{Discussion}

Therapeutic inflammation resulting from different interventions is not a new concept, allowing to generate a tissue repair process. The recent decades have shown a variety of techniques proposed for this therapeutic objective, such as deep transverse massage (Cyriax massage), diacutaneous fibrolysis, therapeutic ultrasound, or extracorporeal shock waves [26-31]. In addition, different invasive procedures for managing diverse musculoskeletal problems as myofascial pain points, muscle contractures, or tendinopathies have become popular.

These percutaneous procedures have the advantage of being more specific for the target tissues, although they require more clinical expertise. MEP is a new therapeutic proposal that uses DC percutaneously through acupuncture needles, and its foundation is also based on the generation of a controlled local inflammatory process accompanied by analgesic effects resulting from the cathode polar effect. MEP is more comfortable than other percutaneous electrical interventions because it uses a microcurrent [8-11, 31].

The objective of this study was to assess the effectiveness of MEP technique in reducing PPT and PI in MTrPs based on the cathode effects that promote a controlled tissue inflammation followed by a regeneration process. This research provided a baseline ultrasound treatment, whose purpose was to safeguard 2 important bioethical principles, justice 
and beneficence, in order to deliver a therapeutic alternative to the group that would not receive MEP once the MTrPs condition was determined. Therapeutic ultrasound was chosen because evidence supported it as a treatment for MTrPs and it has the advantage of not producing sensations of any kind in patients [23]. There were no adverse effects in the participants with both interventions applied. After analysing PPTdiff and PIdiff of the 2 study groups comparing the 3 evaluation sessions, an improvement appears in painful pressure tolerance for algometry test and a decrease in $\mathrm{PI}$ in both groups.

The results show a significant improvement in PPT in the MEP group at the second evaluation session (day 3), which would support the effect of microelectrolysis in a greater increase of painful pressure tolerance. However, this therapeutic advantage compared with therapeutic ultrasound would not be appreciated at the third session and there is no statistically significant difference that allows to affirm that the MEP treatment group was better than the control at 1-week after the intervention. Nevertheless, both groups presented significant improvements in PPT and PI with treatments giving credit to circulatory increase of both applications by breaking muscle spasm cycle of MTrP. Likewise, the good post-inflammatory tissue response induced by MEP would be supported, favouring a faster short-term analgesic effect $[8,10]$.

\section{Limitations}

The recognition of certain limitations is important. As implied by the results, it is necessary to carry out a study with MEP alone to rule out any cumulative effect in favour of ultrasound, although the difference in improvement in the second session in favour of MEP could support its therapeutic effect. Another limitation is related to a small sample size, determined for the convenience of the researchers. It is not clear if the number of participants was enough and if a larger size sample could have resulted in better differences; therefore, it is suggested to determine the sample size statistically in further studies. Another reflection refers to the current dose and number of intervention sessions. The dose $(\mathrm{mA} / \mathrm{min})$ was adjusted in accordance with evidence in the available literature $[3,7]$. It might be possible that different intensities or longer times of treatment would have led to clearer effects; the same applies to more treatment sessions, following the Schultz principle, which indicates that physiological effects achieved are dependent on the magnitude of the applied stimulus [1, 2]. Also, in relation to the treatment dose, the minimum dose necessary to achieve therapeutic effects is not so clear in the microelectrolysis technique. Discomfort thresholds were detected at different emission times in the MEP group participants, so the dose received by each subject varied, reaching different application times. This makes it difficult to standardize an average dose to provide therapeutic results. A statistical analysis of the dose was also performed in the experimental group, giving a mean value of $2.13 \mathrm{~mA} /$ $\min (S D \pm 0.92)$. In that sense, these or similar doses may exhibit differentiating therapeutic effects. Likewise, a greater number of intervention sessions could generate potentiation of physiological effect achieved with the MEP technique, although the work sought to assess whether 1 session could be enough to generate changes in pain. Then, an improvement of our protocol could include more intervention sessions to evaluate whether long-term comparative improvements appear in favour of MEP.

Although the prevalence of MTrPs is not clear or associated with age, it is recognized that the age range of the participants focused more on a young adult population. In that sense, there could be different results in other age ranges with MTrPs presence, which could influence the analgesic response generated by microelectrolysis. The therapeutically obtained effects are clear, but they can also be conditioned by such variables as anxiety or fear of needles and/or electric current, even if not manifested by the participants. That could validly determine people's preferences for non-invasive treatments if the long-term effects are equally favourable.

\section{Conclusions}

This study shows that MEP could be more effective than conventional ultrasound as a treatment of pain in MTrPs in the short term and could be considered as a therapeutic alternative owing to the favourable changes in PPT and PI. Despite not being better than ultrasound in the long term, beneficial changes are also shown in pain reduction of MTrPs. Therefore, the decision to use MEP or other modalities of physical agents such as ultrasound could be subject to the availability of therapeutic resources, the immediacy of the therapeutic effect sought, and the preference of the physiotherapist or the patient receiving the intervention.

It would be interesting to develop more research concerning the use of MEP by improving protocols in relation to dosage modifications, greater number of interventions over time, and using microelectrolysis alone without the synergy of any baseline treatment. It is proposed to continue the research line with microelectrolysis and its results obtained for the improvement of PPT and decrease of PI.

\section{Disclosure statement}

No author has any financial interest or received any financial benefit from this research.

\section{Conflict of interest}

The authors state no conflict of interest.

\section{References}

1. Cameron MH. Physical agents in rehabilitation. St. Louis: Elsevier; 2003.

2. Currier DP, Mann R. Muscular strength development by electrical stimulation in healthy individuals. Phys Ther. 1983;63(6):915-921; doi: 10.1093/ptj/63.6.915.

3. Vance CGT, Dailey DL, Rakel BA, Sluka KA. Using TENS for pain control: the state of the evidence. Pain Manag. 2014;4(3):197-209; doi: 10.2217/pmt.14.13.

4. Martin JMR. Applications and treatment with galvanism [in Spanish]. In: Martin JMR, Electrotherapy in physiotherapy [in Spanish], $3^{\text {rd }}$ ed. Madrid: Editorial Médica Panamericana; 2013; 181-204.

5. Benjamin SJ, Flood JN, Bechtel R, Alon G. Measurement of soft tissue temperature and impedance following the application of transdermal direct current. Physiotherapy. 2007;93(2):114-120; doi: 10.1016/j.physio.2006.11.008.

6. Avendaño Coy J, Ferri Morales A, Sánchez Sobrados E, Ceciaga Ajuria A. Galvanism effects on the excitomotor threshold. A study among healthy subjects [in Spanish]. Rev Iberoam Fisioter Kinesiol. 2001;4(1):32-40.

7. Raiman J, Koljonen M, Huikko K, Kostiainen R, Hirvonen J. Delivery and stability of LHRH and nafarelin in human skin: the effect of constant/pulsed iontophoresis. Eur J Pharm Sci. 2004;21(2-3):371-377; doi: 10.1016/j. ejps.2003.11.003.

8. Delgado A, Ronzio O, da Silva RMV, Soares IJP, da Silva Damasceno RF, Froes PM. Histological analysis of immediate effects caused by percutaneous microelectrolysis 
$\left(\mathrm{MEP}^{\circledR}\right)$ in healthy muscle tissue of Wistar rats [in Portuguese]. ConScientiae Saúde. 2014;13(1):13-21; doi: 10.5585/conssaude.v13n1.4721.

9. Cordero Reyna P, De Benetti C. Eccentric loading versus eccentric loading plus microelectrolisis percutánea (Mep) treatment for mid-portion Achilles tendinopathy: a randomized controlled trial (master thesis). Máster de Fisioterapia del Deporte y Recuperación a la Actividad Física. 2014-2015.

10. Da Silva RMV, de Souza Costa L, do Silva Coldibeli E, do Rosário Soares Fernandes M, Froes Meyer P, Ronzio OA. Effects of Microelectrólisis Percutaneous ${ }^{\circledR}$ on pain and functionality in patients with calcaneal tendinopathy. Rev. MTP Rehab J. 2014;12:185-190; doi: 10.17784/ mtprehabjournal.2014.12.188.

11. Sánchez Ibañez JM. Achilles tendinopathy in an LFP player. Accelerated treatment by means of ultrasoundguided percutaneous intra-tissue electrolysis [in Spanish]. 2008. Available from: https://www.efisioterapia.net/ articulos/tendinopatia-aquiles-un-futbolista-la-Ifptratamiento-acelerado-electrolisis-percutanea-int.

12. Barbero M, Schneebeli A, Koetsier E, Maino P. Myofascial pain syndrome and trigger points: evaluation and treatment in patients with musculoskeletal pain. Curr Opin Support Palliat Care. 2019;13(3):270-276; doi: 10.1097/ SPC.0000000000000445.

13. Behm DG, Wilke J. Do self-myofascial release devices release myofascia? Rolling mechanisms: a narrative review. Sports Med. 2019;49(8):1173-1181; doi: 10.1007/ s40279-019-01149-y.

14. Dommerholt J, Chou L-W, Finnegan M, Hooks T. A critical overview of the current myofascial pain literature February 2019. J Bodyw Mov Ther. 2019;23(2):295-305; doi: 10.1016/j.jbmt.2019.02.017.

15. Fricton J. Myofascial pain: mechanisms to management. Oral Maxillofac Surg Clin North Am. 2016;28(3):289-311; doi: 10.1016/j.coms.2016.03.010.

16. Palacios-Ceña M, Wang K, Castaldo M, Guerrero-Peral Á, Caminero AB, Fernández-de-Las-Peñas $C$, et al. Assessment of deep dynamic mechanical sensitivity in individuals with tension-type headache: the dynamic pressure algometry. Eur J Pain. 2017;21(8):1451-1460; doi: 10.1002/ejp.1065.

17. Uddin Z, MacDermid JC. Quantitative sensory testing in chronic musculoskeletal pain. Pain Med. 2016;17(9): 1694-1703; doi: 10.1093/pm/pnv105.

18. Nussbaum EL, Downes L. Reliability of clinical pressurepain algometric measurements obtained on consecutive days. Phys Ther. 1998;78(2):160-169; doi: 10.1093/ptj/ 78.2.160.

19. Kinser AM, Sands WA, Stone MH. Reliability and validity of a pressure algometer. J Strength Cond Res. 2009;23(1): 312-314; doi: 10.1519/JSC.0b013e31818f051c.

20. Espejo-Antúnez L, Fernández-Huertas Tejeda J, Albornoz-Cabello M, Rodríguez-Mansilla J, de la Cruz-Torres B, Ribeiro F, et al. Dry needling in the management of myofascial trigger points: a systematic review of randomized controlled trials. Complement Ther Med. 2017;33: 46-57; doi: 10.1016/j.ctim.2017.06.003.

21. Hong $\mathrm{C}-\mathrm{Z}$. Algometry in evaluation of trigger points and referred pain. J Musculoskelet Pain. 1998;6(1):47-59; doi: 10.1300/j094v06n01_04.

22. Heller GZ, Manuguerra M, Chow R. How to analyze the Visual Analogue Scale: myths, truths and clinical relevance. Scand J Pain. 2016;13:67-75; doi: 10.1016/j. sjpain.2016.06.012.
23. Xia P, Wang X, Lin Q, Cheng K, Li X. Effectiveness of Ultrasound therapy for myofascial pain syndrome: a systematic review and meta-analysis. J Pain Res. 2017;10: 545-555; doi: 10.2147/JPR.S131482.

24. Linde LD, Kumbhare DA, Joshi M, Srbely JZ. The relationship between rate of algometer application and pain pressure threshold in the assessment of myofascial trigger point sensitivity. Pain Pract. 2018;18(2):224-229; doi: 10.1111/papr.12597.

25. Pitsillides A, Stasinopoulos D. Cyriax friction massage suggestions for improvements. Medicina. 2019;55(5): 185; doi: 10.3390/medicina55050185.

26. Joseph MF, Taft K, Moskwa M, Denegar CR. Deep friction massage to treat tendinopathy: a systematic review of a classic treatment in the face of a new paradigm of understanding. J Sport Rehabil. 2012;21(4):343-353; doi: 10.1123/jsr.21.4.343.

27. Jiménez Del Barrio S, Estébanez de Miguel E, Bueno Gracia E, Haddad Garay M, Tricás Moreno JM, Hidalgo García C. Effects of diacutaneous fibrolysis in patients with mild to moderate symptomatic carpal tunnel syndrome: a randomized controlled trial. Clin Rehabil. 2018; 32(12):1645-1655; doi: 10.1177/0269215518787316.

28. Liao C-D, Xie G-M, Tsauo J-Y, Chen H-C, Liou T-H. Efficacy of extracorporeal shock wave therapy for knee tendinopathies and other soft tissue disorders: a metaanalysis of randomized controlled trials. BMC Musculoskelet Disord. 2018;19(1):278; doi: 10.1186/s12891018-2204-6.

29. Zhang L, Fu X-B, Chen S, Zhao ZB, Schmitz C, Weng C-S. Efficacy and safety of extracorporeal shock wave therapy for acute and chronic soft tissue wounds: a systematic review and meta-analysis. Int Wound J. 2018;15(4):590599; doi: 10.1111/iwj.12902.

30. Zhang C, Xie Y, Luo X, Ji Q, Lu C, He C, et al. Effects of therapeutic ultrasound on pain, physical functions and safety outcomes in patients with knee osteoarthritis: a systematic review and meta-analysis. Clin Rehabil. 2016;30(10):960-971;doi:10.1177/0269215515609415.

31. De la Barra Ortiz HA, Opazo J, Romero Poblete I, Mora Santis J. Effects of cathode and anode of the direct current on changes in palmar grip strength: assessment through a dynamometry. Fisioter Pesqui. 2018;25(1): 115-123; doi: 10.1590/1809-2950/17460125012018. 